OPEN ACCESS

Edited by:

Ivan Mijakovic,

Chalmers University of Technology,

Sweden

Reviewed by:

Marie-Françoise Noirot-Gros, Argonne National Laboratory, USA

Anthony Baughn,

University of Minnesota, USA

*Correspondence:

Thomas Dick

thomas_dick@nuhs.edu.sg

Specialty section:

This article was submitted to

Microbial Physiology and Metabolism,

a section of the journa

Frontiers in Microbiology

Received: 29 June 2016 Accepted: 23 August 2016

Published: 31 August 2016

Citation:

Wu M-L, Chan CL and Dick T (2016) Rel Is Required for Morphogenesis of Resting Cells in Mycobacterium smegmatis. Front. Microbiol. 7:1390 doi: 10.3389/fmicb.2016.01390

\section{Rel Is Required for Morphogenesis of Resting Cells in Mycobacterium smegmatis}

\author{
Mu-Lu Wu, Chuu Ling Chan and Thomas Dick* \\ Antibacterial Drug Discovery Laboratory, Department of Microbiology and Immunology, Yong Loo Lin School of Medicine, \\ National University of Singapore, Singapore, Singapore
}

Recently we showed that upon transfer of growing Mycobacterium smegmatis into saline, the bacilli exited the canonical cell division cycle and formed septated multinucleoided cells. Under shock starvation (i.e., in saline without any carbon source), differentiation terminated at this stage with internally remodeled Large Resting Cells (LARCs). Whereas under gentle starvation (i.e., in saline with trace amounts of a carbon source), the septated multi-nucleoided bacilli completed cell division and separated into mono-nucleoided Small Resting Cells (SMRCs). This demonstrated that the non-sporulating mycobacteria are in fact capable of forming morphologically differentiated resting cells when exposed to starvation. Depending on the specific starvation conditions they can form two different resting cell types, LARCs or SMRCs, which share a common cellular differentiation pathway. The mRNA encoding the (p)ppGpp synthetase Rel was found to be transiently upregulated immediately upon starvation under both conditions, suggesting a role for the stringent response factor in both LARC and SMRC development. Here, we disrupted Rel function by generating two types of mutant $M$. smegmatis strains: a rel nonsense mutant (re/E4TAG) in which translation is prematurely terminated at codon 4 , and a rel deletion mutant ( $\Delta$ rel) in which the entire coding sequence was deleted. Both mutants showed identical phenotypes: sparse septum formation, less DNA compaction, and failure in formation of both the septated multi-nucleoided LARCs and the small-cell morphotype SMRC under starvation conditions. All phenotypes were rescued through the introduction of a wildtype copy of rel. Therefore, we conclude that loss-of-function mutations in rel block the development of both LARCs and SMRCs by preventing the first morphogenetic step in mycobacterial resting cell development, the formation of septated multi-nucleoided cells. Interestingly, in contrast to Rel's role in most other bacteria, starvation survival was not affected by loss of rel function. Our results suggest that Rel may play a starvation-induced morphogenetic role in mycobacteria.

Keywords: stringent response, starvation, bacterial differentiation, SMRC, LARC

\section{INTRODUCTION}

Bacteria are constantly exposed to nutrient limitations in their natural environments (Morita, 1993; Rittershaus et al., 2013). To endure these harsh conditions, most bacteria activate a broadly conserved starvation stress response called the stringent response (Potrykus and Cashel, 2008; Dalebroux and Swanson, 2012; Boutte and Crosson, 2013). Upon nutrient deprivation, 
the concentration of the signaling nucleotides guanosine tetraphosphate and guanosine pentaphosphate [(p)ppGpp] is rapidly increased by the (p)ppGpp synthetase, RelA/SpoT homologue proteins (RSHs). The elevated cellular concentration of (p)ppGpp alters the transcription of a wide range of genes, thus mediating the physiological adaptation to nutrient starvation and ensuring bacterial survival (Hauryliuk et al., 2015). The stringent response was originally discovered by Stent and Brenner (1961) in Escherichia coli. Later it was found to be critical across many bacterial phyla (Braeken et al., 2006; Boutte and Crosson, 2013; Hauryliuk et al., 2015), including mycobacteria (Ojha et al., 2000; Primm et al., 2000; Mathew et al., 2004; Dahl et al., 2005). In mycobacteria, the amount of (p)ppGpp under starvation is mainly modulated by the bifunctional RSH protein Rel which synthesizes and hydrolyzes (p)ppGpp (Avarbock et al., 1999, 2005; Jain et al., 2006). Disruptions of rel in mycobacteria were reported to result in defective survival under starvation in vitro and loss of virulence in animal infection models (Primm et al., 2000; Dahl et al., 2003, 2005; Klinkenberg et al., 2010).

Mycobacteria are capable of retaining viability under starvation in pure saline for extended periods of time by entering a non-replicating state accompanied by reduced metabolism and increased phenotypic drug resistance, but without any apparent morphological differentiation (Loebel et al., 1933a,b; Nyka, 1974; Betts et al., 2002; Xie et al., 2005; Gengenbacher et al., 2010). Recently, we reported that gentle starvation of Mycobacterium smegmatis in saline (PBS) containing traces of a carbon source triggered the development of mono-nucleoided Small Resting Cells (SMRCs), with septated multi-nucleoided cells as intermediates. In contrast, shock starvation in zeronutrient PBS resulted in apparently unaltered log phase-sized resting cells. Surprisingly, fluorescence microscopic analyses revealed that these shock starved 'normal sized' bacilli had remodeled their interior to form septated multi-nucleoided resting cells which we termed Large Resting Cells (LARCs; Wu et al., 2016). Comparative developmental transcriptome analyses showed an early and transient upregulation of the rel mRNA under both gentle and shock starvation (Wu et al., under review), suggesting that the mycobacterial (p)ppGpp synthetase may play a role in both LARC and SMRC formation.

In this study, to investigate the function of Rel in nutrient deprivation for M. smegmatis, rel mutants were constructed. We showed that loss-of-function mutations in rel blocked the development of both LARCs and SMRCs by preventing the formation of septated multi-genomic bacilli, the first cellular differentiation step in mycobacterial resting cell development.

\section{MATERIALS AND METHODS}

\section{Bacterial Strains and Culture Conditions}

Mycobacterium smegmatis $\mathrm{mc}^{2} 155$ (ATCC 700084) was cultivated at $37^{\circ} \mathrm{C}$ with agitation in Middlebrook $7 \mathrm{H} 9$ broth containing $0.5 \%$ bovine albumin, $0.2 \%$ glucose, $0.085 \% \mathrm{NaCl}$, $0.5 \%$ glycerol, $0.0003 \%$ catalase, and $0.05 \%$ Tween 80 . In nutrient starvation experiments, mid-log-phase cultures with an optical density at $600 \mathrm{~nm}\left(\mathrm{OD}_{600}\right)$ of 0.5 were harvested by centrifugation $\left(3200 \mathrm{rpm}, 10 \mathrm{~min}, 25^{\circ} \mathrm{C}\right.$ ) and washed three times with PBS-0.025\% Tween 80 or PBS- $0.025 \%$ Tyloxapol (PBS, Gibco 14080-055). The cultures were then diluted in the corresponding starvation media to a final $\mathrm{OD}_{600}$ of $0.10-0.15$, and subsequently transferred to a 1-1 roller bottle (Corning, COR430195). The starved cultures were incubated for 14 days with rolling at $2 \mathrm{rpm}$ at $37^{\circ} \mathrm{C}$. To determine CFUs, appropriate dilutions of cultures were plated on Middlebrook $7 \mathrm{H} 10$ agar plates supplemented with $0.5 \%$ bovine albumin, $0.2 \%$ glucose, $0.085 \% \mathrm{NaCl}, 0.5 \%$ glycerol, $0.0003 \%$ catalase, and $0.006 \%$ oleic acid, and colony numbers were numerated after 2 days incubation.

\section{Staining and Microscopy}

Acid-fast staining was carried out using a TB stain kit (BD, 212520) according to manufacturer's instructions and observed under a light microscope (Olympus BX60, brightfield). For FM464, DNA and Nile red staining, samples were collected at 14 days by centrifugation and then fixed with $4 \%$ paraformaldehyde in PBS for $30 \mathrm{~min}$. Fixed samples were then stained and viewed as previously described (Wu et al., 2016). For live/dead staining, LIVE/DEAD BacLight bacterial viability kit (Molecular Probes L7012) was used according to manufacturer's manual. Briefly, collected live cultures were washed and resuspended in $0.85 \%$ $\mathrm{NaCl}$ solutions, followed by incubation with equal and proper amount of propidium iodide and Styo 9 in the dark for $15 \mathrm{~min}$. Stained samples were then mounted to slides and observed under epifluorescence microscope Olympus BX60 using the U-MWIG and U-MWIB filters for propidium iodine and Styo, respectively.

\section{Construction of relE4TAG, $\Delta r e l$, and complemented strains}

DNA and mycobacterial genetic manipulations were carried out as described previously (Kaser et al., 2009). The rel nonsense mutant $r l^{\mathrm{E} 4 \mathrm{TAG}}$ was generated by introducing stop codon TAG at the fourth triplet of the rel coding sequence (MSMEG_2965) using the point mutation recombineering protocol described by van Kessel and Hatfull (2008). Single-stranded DNA oligos (5'ACCGCGGGAGGAGACTGCACGGCCTGACCCTTGCCTGG CTAGGTCGACCATGGTTGTCACCTCCTGCCCAGCAACCCG AATT- $3^{\prime}$ ) harboring the desired point mutation at the center were introduced into electrocompetent $M$. smegmatis containing pJV62. Positive mutants were screened using PCR amplification (5' - GTGACAACCATGGTCGACT-3' and 5' -TTCGGGTAGAT CTCGCGGT- $3^{\prime}$ ) and verified by DNA sequencing (5' - CCGA AGGAATTGACATCGC-3', and 5'-TTCGGGTAGATCTCGC GGT-3'; Supplementary Figure 1A). Deletion of the rel coding sequence was carried out by gene replacement recombineering method as described in van Kessel et al. (2008). A 500 bp fragment upstream of rel was amplified using primers $5^{\prime}$ ACGTCTTAAGCCAGTTCAAGGACCTCACGCC-3' and $5^{\prime}$ CTAGTCTAGAGGTTGTCACCTCCTGCCCAG-3'. Similarly, a 513 bp fragment downstream of rel (including the last six nt in rel coding sequence) was amplified using $5^{\prime}$ - CTCG AAGCTTGCCTGATCGAGCGGTTCGGCGT- $3^{\prime}$ and $5^{\prime}$ - CTAG ACTAGTCTCCTCGTGGACAGGATCGGCCGA-3'. These two 
fragments were sequentially cloned into vector pYUB854 flanking the hygromycin resistance cassette at AflII/XbaI and HindIII/SpeI sites, respectively, to generate pYUB854-relKO. $2.9 \mathrm{~kb}$ double-stranded allelic exchange substrates obtained by digesting pYUB854-relKO with AflII and SpeI-HF were electroporated into $M$. smegmatis containing vector pJV53 to generate rel knockout strain $\Delta$ rel. The complete rel coding sequence (except the last six nt) was verified to be replaced by the hygromycin ${ }^{R}$ cassette via PCR analysis (Supplementary Figures 1B,C) and DNA sequencing. For complementation, the rel coding sequence from Mycobacterium tuberculosis (Rv2583c) including the $260 \mathrm{bp}$ upstream containing the native rel promoter was isolated via PCR amplification with the primer set $5^{\prime}$ CTCGAAGCTTACGGCGCCGCCACTCTGGAGATTC- $3^{\prime}$ and 5' - ACGTGGTACCCTACGCGGCCGAGGTCACCCGGTA-3', and cloned into the HindIII and KpnI sites of the integrative mycobacterial shuttle vector pMV306 to generate plasmid pMV306-Rel $\mathrm{Mtb}_{\mathrm{Mtb}}$. The $\mathrm{rel}_{\mathrm{Mtb}}$ sequence in the complementation construct pMV306-rel $l_{\text {Mtb }}$ was verified by DNA sequencing. Successful integration of $M$. tuberculosis rel allele into the $\Delta$ rel background was verified by positive PCR amplification with primer set $5^{\prime}$-ACAACATGCGCACCATGCG-3' and $5^{\prime}$ CAAGCGCTGCAACGGAAGTC-3' (Supplementary Figure 1C, lane 3). Due to the high homology of rel alleles between $M$. tuberculosis and M. smegmatis, this set of primers binds to both wild-type $M$. smegmatis DNA and the $\Delta$ rel complemented strain ( $\Delta$ relComp) that contains the $M$. tuberculosis rel copy.

\section{RESULTS}

\section{Rel-Disrupted M. smegmatis Mutants Fail to Form SMRCs under Gentle Starvation}

To determine the role of Rel in mycobacterial resting cell differentiation, we introduced a TAG stop codon at the fourth triplet of the rel coding sequence via oligonucleotide-based site directed genome mutagenesis ('recombineering') to block synthesis of the protein (see Material and Methods for details). The verified rel nonsense mutant, named $\mathrm{rel}^{\mathrm{E} 4 \mathrm{TAG}}$, was then subjected to growth in nutrient broth, shock starvation in PBS, or mild starvation in PBS containing traces of Tween80, a fatty acid ester used by mycobacteria as a carbon source (Tomioka, 1983). Figure 1A (rel I $\left.4 \mathrm{TAG}^{2}\right)$ shows that the rel nonsense mutation did not affect the growth rate or cell shape of M. smegmatis in nutrient broth (Gupta et al., 2016), but the
A
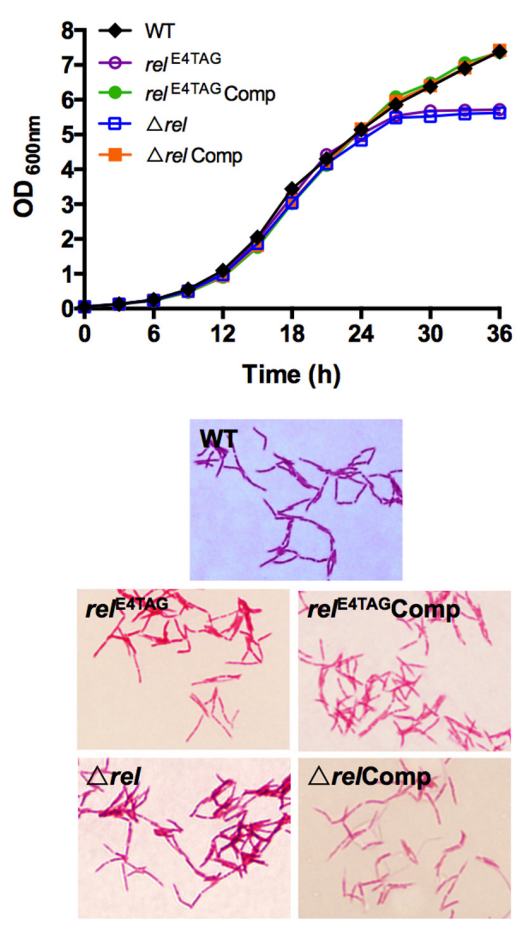

B
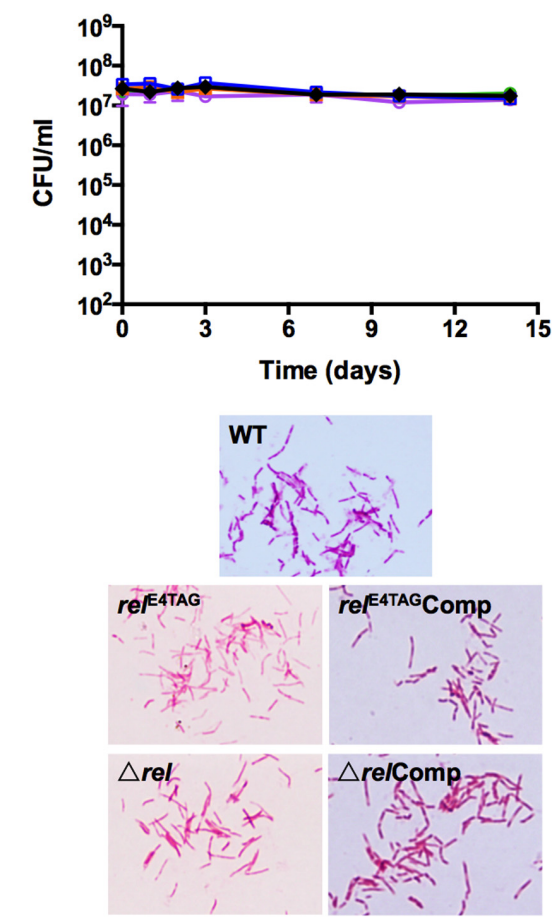

C

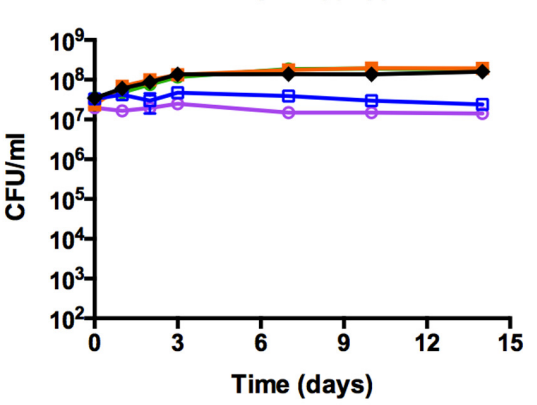

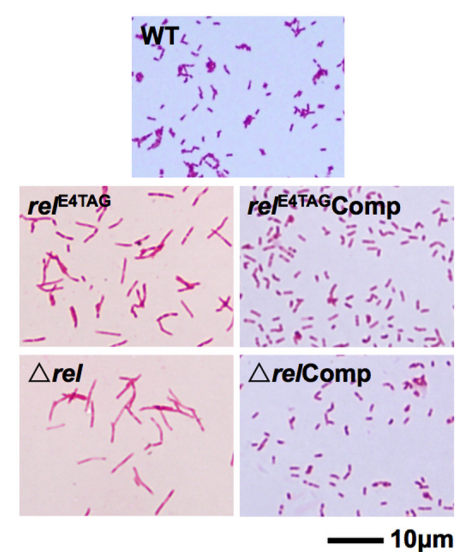

FIGURE 1 | Growth, starvation survival, and cell size phenotypes of Mycobacterium smegmatis re ${ }^{\text {E4TAG }}$ and $\Delta$ rel. (A) Growth curves and acid-fast stained log-phase culture samples of $M$. smegmatis re ${ }^{E 4 T A G}$ and $\Delta$ rel in nutrient broth in comparison with wild-type $M$. smegmatis and the two complemented mutant strains (re/E4TAG Comp and $\Delta$ re/Comp). (B,C) Survival curves and acid-fast stained 14-day starved samples of $M$. smegmatis relE4TAG and $\Delta$ rel in (B) PBS and (C) PBS-Tween80 in comparison with wild-type M. smegmatis and the two complemented strains. In all curves, black diamond: wild-type strain; purple empty circle:

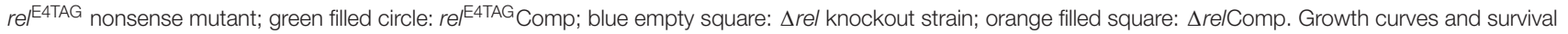
curves data are shown as means \pm standard deviations from two independent biological replicates. Representative fields were chosen for acid-fast stained images. 
mutant strain entered into stationary phase earlier than wildtype bacilli. Surprisingly, the rel nonsense mutation did not affect survival of the bacilli when subjected to starvation conditions (Figures 1B,C). Microscopic analyses of acid-fast stained samples depicted in Figure 1C showed that the $M$. smegmatis rel nonsense mutant grown under gentle starvation conditions (PBSTween 80 ) failed to form SMRCs. Only $8 \%$ of cells in starved rel nonsense mutant cultures were of the SMRC morphotype, whereas $95 \%$ of cells in wild-type cultures were SMRCs $(n=100)$, suggesting a role of Rel in the formation of the small-cell morphotype. Consistent with this microscopic observation, the slight increase in CFU observed for wild-type bacteria under gentle starvation due the separation of the septated multinucleoided cell intermediates into mono-nucleoided SMRCs, was not observed for $M$. smegmatis rel l4TAG $^{\mathrm{E} \text { (Figure 1C). Both }}$ the microscopic and the CFU phenotype could be restored by introduction of a wild-type allele of $M$. tuberculosis rel under its native promoter into the $M$. smegmatis rel nonsense mutant background (Figure 1C, rel ${ }^{\mathrm{E} 4 \mathrm{TAG}} \mathrm{Comp}$ ). No apparent cell size difference was observed in acid-fast stained rel nonsense mutants in PBS starved cultures (Figure 1B, rel ${ }^{\mathrm{E} 4 \mathrm{TAG}}$ ). Taken together, analyses of a rel nonsense mutant suggest that Rel is required for the formation of SMRCs.

To exclude the possibility that the rel nonsense mutant may be leaky, i.e., allows some translational read-through of the stop codon, we generated a $M$. smegmatis $\Delta$ rel strain in which the entire coding sequence of rel was deleted and replaced with a hygromycin resistance cassette (see Material and Methods for details). Figure 1 ( $\Delta r e l$ ) shows that all the phenotypes displayed by the $\Delta$ rel loss-of-function mutant were identical to that observed for the rel nonsense mutant, demonstrating that the introduced stop codon in the nonsense mutant effectively blocked expression of the Rel product. As shown for the rel nonsense mutant, deletion of rel blocked the formation of SMRCs (only 5\% of $\Delta$ rel mutants formed small cells, $n=100$ ) and this phenotype was rescued by complementation with the wild-type allele of the gene (Figure 1C, $\Delta$ relComp).

\section{rel Loss-of-Function Mutation Blocks the First Step in Mycobacterial Resting Cell Formation - The Generation of Septated Multi-nucleoided Cells}

The acid-fast staining results showed that $M$. smegmatis rel loss-of-function strains failed to form SMRCs in gently starved cultures (Figure 1C). To determine whether loss of rel function affects the formation of the LARC-like septated, multi-nucleoided intermediates during SMRC development and/or the formation of LARCs under conditions of shock starvation, we carried out fluorescence microscopic analyses on M. smegmatis $\Delta r e l$ cultures subjected to gentle PBS-Tween80

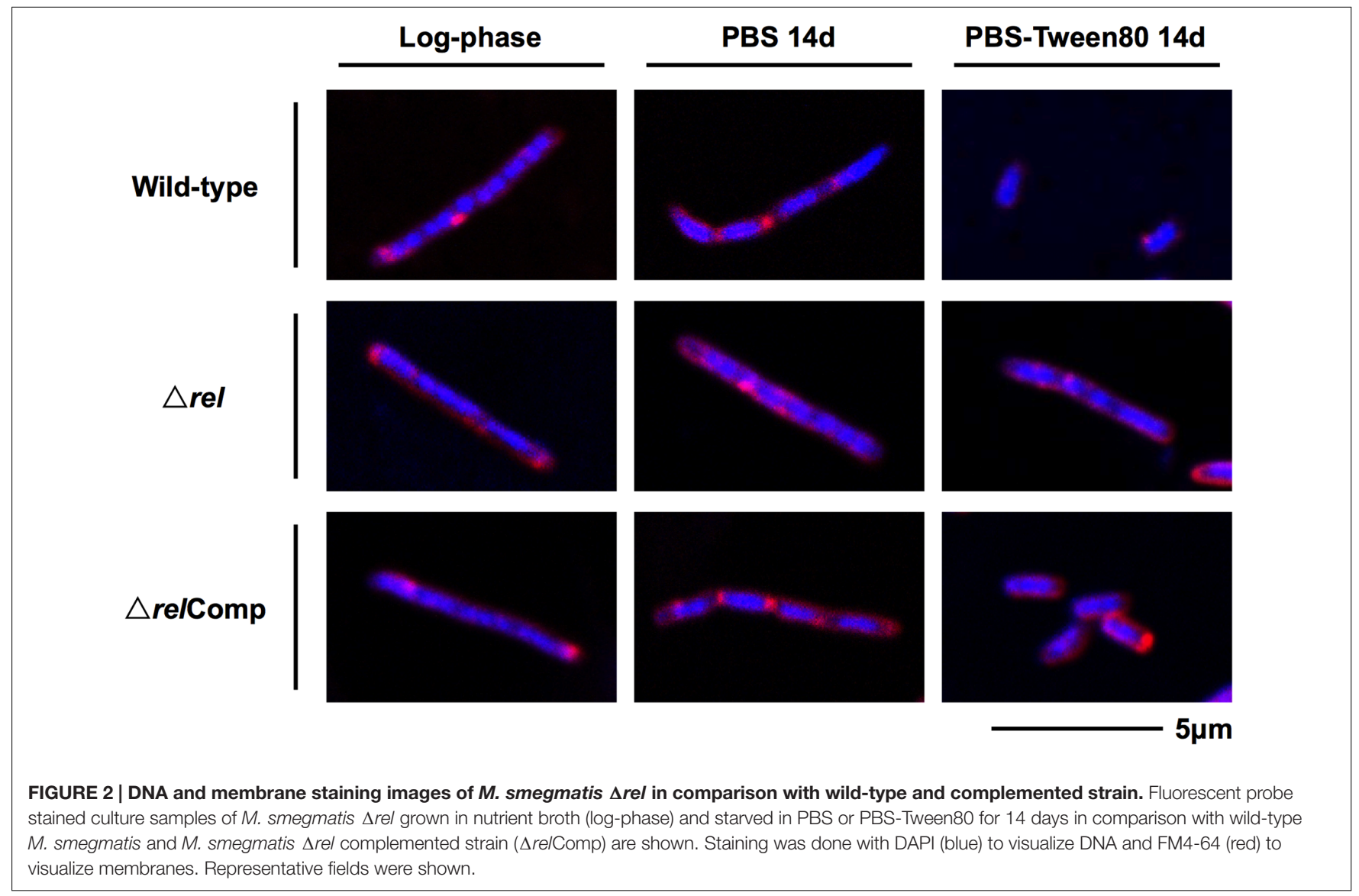




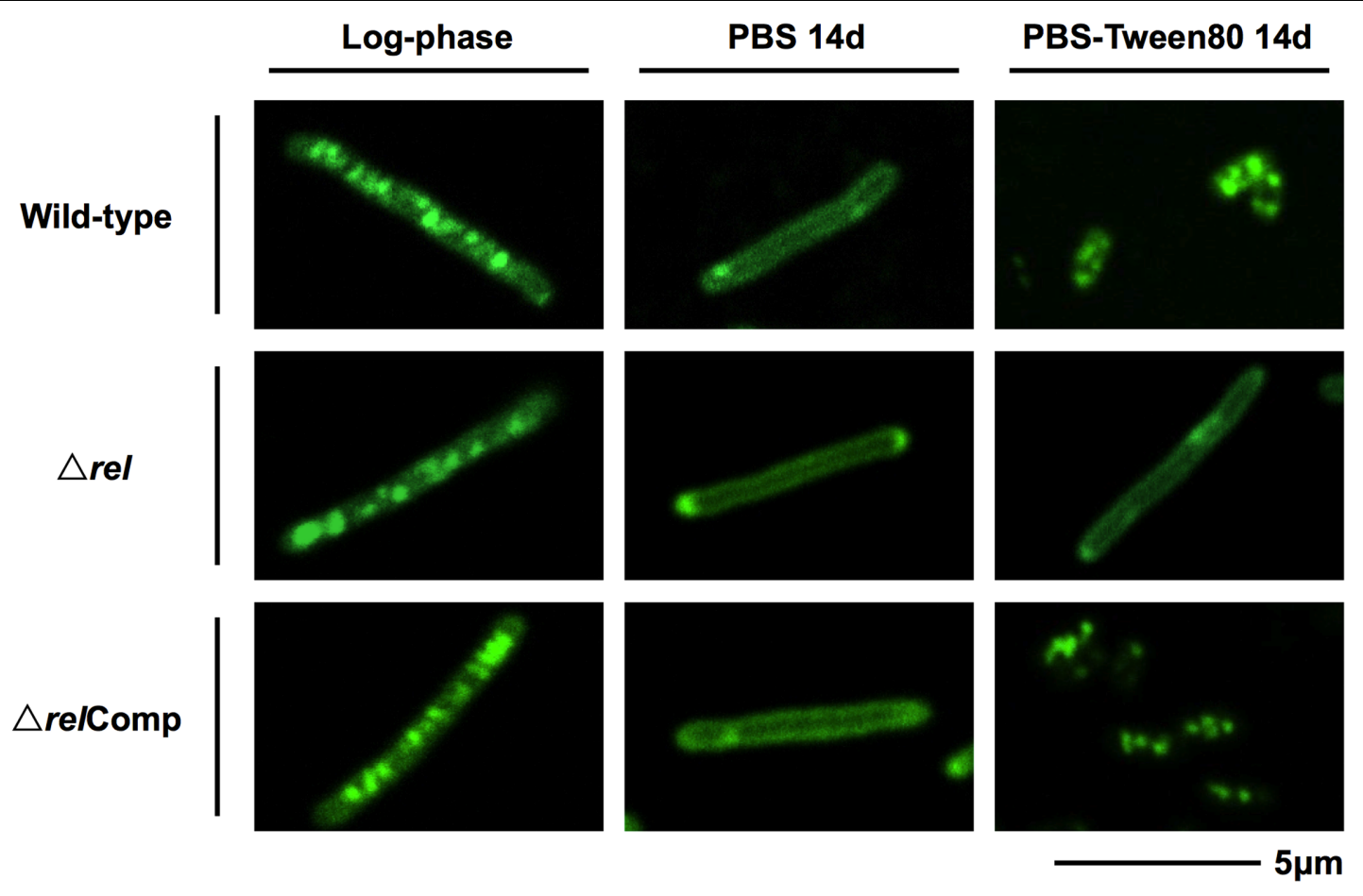

FIGURE 3 | Lipid body staining images of $\boldsymbol{M}$. smegmatis $\Delta$ rel in comparison with the wild-type and complemented strain. Fluorescent probe stained culture samples of $M$. smegmatis $\Delta$ rel grown in rich broth (log-phase) and starved in PBS or PBS-Tween80 for 14 days in comparison with wild-type M. smegmatis and M. smegmatis $\Delta$ rel complemented strain ( $\Delta$ re/Comp) are shown. Staining was done with Nile red (green) to visualize intracellular lipid bodies. Nile red stains predominantly intracellular lipid inclusions. Representative fields were shown.

and shock PBS starvation. Starved cultures were stained with the fluorescent probes DAPI and FM4-64 to visualize DNA and membrane structure, respectively. As reported in our previous study (Wu et al., 2016), around 70\% of wild-type M. smegmatis cells formed septated multi-nucleoided bacilli (containing 1-3 septa per cell) under shock starvation. In contrast, Figure 2 shows that $\Delta$ rel bacilli failed to form septated cells in PBS. Only sparse septum formation (none or one) was observed in shock-starved $\Delta$ rel bacilli: $72 \%$ of the cells did not contain any septum and $26 \%$ showed one septum $(n=70)$. Likewise, in PBS-Tween80 cultures where the majority of the wild-type cells produced SMRCs via a septated multi-nucleoided intermediate stage (Wu et al., 2016), few septated multi-nucleoided cells were observed: $66 \%$ of $\Delta$ rel bacilli did not contain any septum while $27 \%$ had only one septum $(n=100)$. In addition to the septation pattern, DNA segregation and compaction were also affected by the absence of Rel protein. In contrast to the highly condensed nucleoids observed in starved wild-type cells, DNA in $\Delta$ rel bacilli distributed throughout the whole cell under both starvation conditions, i.e., the DNA was less condensed (Figure 2). Furthermore, loss of rel function resulted in the loss of intracellular lipid bodies in PBS-Tween80 starved cultures (Figure 3). Unexpectedly, we observed additional cell structures in PBS shock-starved rel mutant cultures upon DAPI and Nile red staining and these structures will be discussed below. Analyses of the M. smegmatis loss-of-function strain complemented with the wild-type allele of rel restored septum formation, DNA compaction and intracellular lipid body accumulation phenotypes (Figures 2 and 3, $\Delta$ relComp). These results indicate that Rel is required for both LARC and SMRC formation and that loss-of-function of rel blocks the first cellular step of mycobacterial resting cell formation - the generation of septated multi-nucleoided cells. This genetic function of rel is consistent with the observation that the rel gene is transiently upregulated under both culture conditions prior to the onset of septation and nucleoid compaction ( $\mathrm{Wu}$ et al., under review).

It is interesting to note that during fluorescence microscopic analyses using DAPI or Nile red stain, additional cell morphotypes were observed specifically in 14-day old PBS shock starved $M$. smegmatis $\Delta$ rel cultures. These novel cell structures, either containing a ball-like attachment (Figure 4, upper row) or being ball-like (Figure 4, lower row), were comparable in number to the number of 'intact' acidfast positive bacilli described above under these culture conditions. Notably, these ball-like structures were not observable in acid-fast stained samples, suggesting that they have an altered cell wall thus affecting the staining behavior. These structures were also only weakly stained by FM4-64. Moreover, these structures were permeable to propidium iodide (Figure 4D), indicating a breakdown of the 

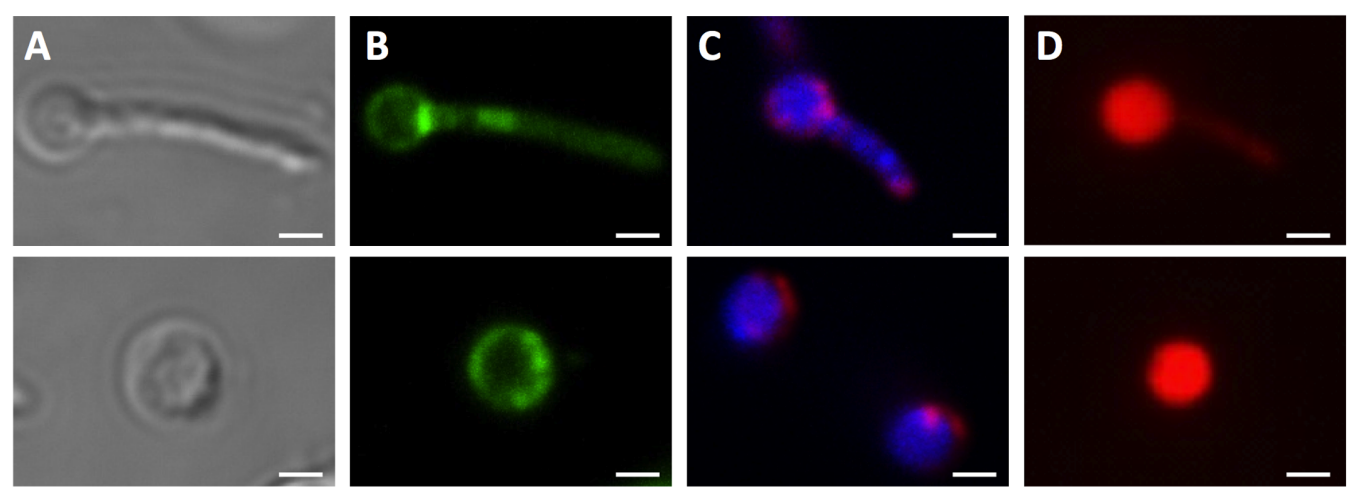

FIGURE 4 | Unusual cell shapes in PBS-starved cultures of $\boldsymbol{M}$. smegmatis $\boldsymbol{\Delta}$ rel. Typical examples of unusual 'ball-like' cell shapes ( 60\%) observed in 14-day PBS-starved M. smegmatis $\Delta$ rel cultures are shown. Cells carried ball-like appendices are shown in the upper panels, and ball-shaped cells are shown in the lower panels. (A) Differential interference contrast images to visualize cell shapes. (B) Nile red (green) stained samples to visualize lipid bodies. (C) DAPI (blue) and FM4-64 (red) double stained samples to visualize DNA and membrane, respectively. (D) Propidium iodide (red) staining was done to show breakdown of the permeability barrier of the ball-like structures. These structures were observed under phase contrast but not in acid-fast stained samples, suggesting that these structures may be cell wall defective. Representative fields were shown. White bar $=1 \mu \mathrm{m}$.

cell permeability barrier. It remains to be determined whether these DNA-containing structures are dead or injured cells with compromised cell permeability, as propidium iodide stained cells do not necessarily represent dead cells (Shi et al., 2007).

\section{DISCUSSION}

Recently, we showed that "non-sporulating" M. smegmatis is in fact capable of forming morphologically distinct resting cells. Gentle starvation in PBS with traces of a carbon source triggers the development of mono-nucleoided SMRCs, with septated multi-nucleoided cells as intermediates. Shock starvation in PBS with zero carbon source results in the formation of LARCs, which are similar in shape and size to log phase cells. However, compared to growing bacilli, LARCs show internal remodeling to form septated multi-nucleoided cells, similar to the intermediates observed during SMRC development. Based on these observations, we proposed that M. smegmatis harbors a novel starvation-induced morphological differentiation program: Upon nutrient limitation, bacilli exit the canonical cell division cycle and form septated multinucleoided cells. Under zero-nutrient conditions, the bacilli terminate development at this stage as LARCs. But in the presence of traces of a carbon source, these multi-nucleoided cells continue differentiation. They complete cell division and separate into mono-nucleoided SMRCs (Figure 5A; Wu et al., 2016). To begin to dissect the genetic program underlying SMRC/LARC differentiation, we generated loss-of-function mutations in one of the early and transiently upregulated genes common to SMRCs and LARCs, the stringent response factor rel. Fluorescence microscopic analyses showed that the rel mutations prevented the first cellular differentiation step in SMRC/LARC formation - the generation of septated multigenomic cells with condensed chromosomes (Figure 5A).
Interestingly, starvation survival of the bacilli was not affected in rel loss-of-function strains, suggesting a role for the mycobacterial Rel in starvation-induced morphogenesis rather than in starvation survival.

\section{Stringent Response vs. Starvation Survival}

The lack of a starvation survival phenotype for our loss-offunction mutants was surprising because it was previously reported that a $M$. smegmatis rel mutant showed $\sim 2-\log$ reduction of CFU after 28-day starvation in Tris-buffered salineTween80 (TBST; Dahl et al., 2005). However, we did not observe any loss of viability in our $M$. smegmatis $\Delta$ rel mutant. To investigate this difference, the nutrient starvation experiments were repeated in TBST, but we observed the same results as with PBS whereby there was no loss of viability (data not shown). The reason for the discrepancy remains to be determined. However, it was noted that the authors only deleted part of the coding sequence, the (p)ppGpp synthetase domain, leaving the (p)ppGpp hydrolase domain of Rel intact (Dahl et al., 2005).

Recently, in various bacterial species, small alarmone synthetases (SASs) - small proteins which contain only the synthetase domain - have been discovered as alternative contributors to the cellular (p)ppGpp level (Hauryliuk et al., 2015). In 2012, Murdeshwar and Chatterji (2012) reported the presence of a second monofunctional (p)ppGpp synthetase in the M. smegmatis genome. This short alarmone synthetase, named MS_RHII-RSD, was reported to constitutively produce a basal level of (p)ppGpp in the exponential growth phase, as well as under starvation and other stress conditions, including oxidative stress, osmotic stress, and acidic stress (Murdeshwar and Chatterji, 2012). An alternative source of (p)ppGpp provided by such SASs has been shown to contribute to bacterial metabolic balance and stress tolerance in other bacterial species, e.g., Enterococcus faecalis, Vibrio 
A

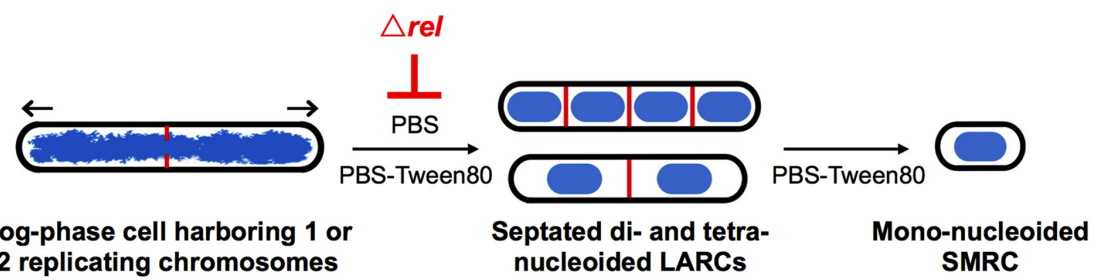

B

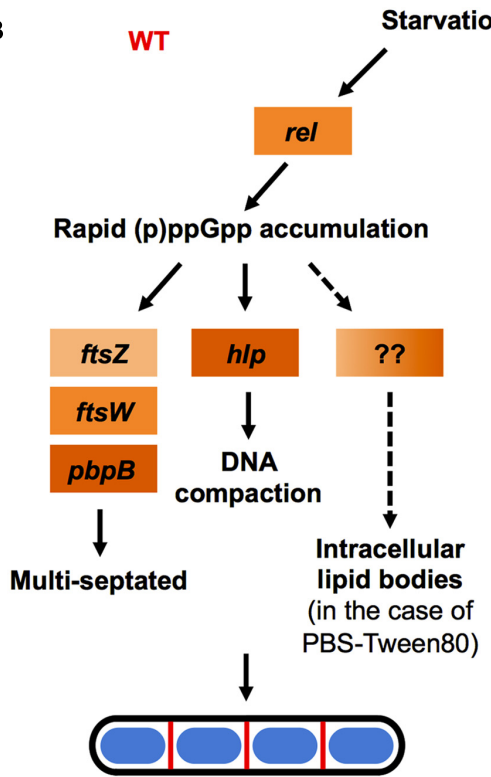

PBS-Tween80

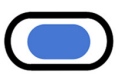

(mild/shock)

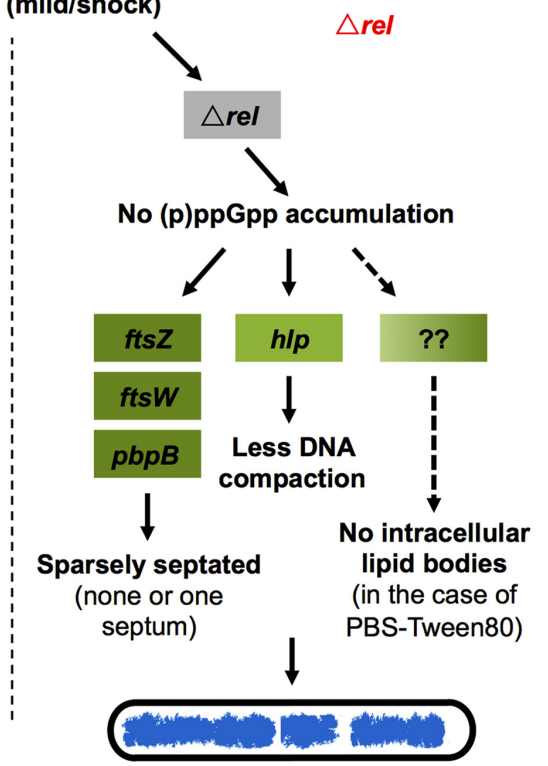

\begin{tabular}{l|l|l|l|l|l|}
-5 & -4 & -3 & 3 & 4 & 5
\end{tabular}

Fold change

FIGURE 5 | Schematic of Rel's potential roles in SMRC (Small Resting Cell)/LARC (Large Resting Cell) development. (A) Model depicting the nutrient starvation induced cellular differentiation program in M. smegmatis. Loss of rel function blocks the formation of septated multi-nucleoided cells seen as LARCs or the intermediates in SMRC formation. Blue: DNA, red: septa, black: cell envelope. Arrows indicate polar growth of the replicating log-phase bacilli. (B) Model on how Rel may be involved in the formation of the first cellular differentiation step, the formation of septated, multi-genomic cells with condensed nucleoids. For the left panel, expression changes are shown as fold changes of $3 \mathrm{~h}$-starved cultures compared with log-phase cells as described in Wu et al. (under review). Orange colors indicate increase in transcript levels. For the right panel, expression changes of $f t s W, p b p B$, and $h / p$ are shown as fold changes of $6 \mathrm{~h}$-starved $\Delta$ rel mutant vs. 6 h-starved wild-type Mycobacterium tuberculosis as reported by Dahl et al. (2003), while the transcript level of fts $Z$ is shown as fold change of starved $\Delta$ rel mutant vs. starved wild type M. smegmatis as reported by Gupta et al. (2016). Green colors indicate decrease in transcript levels.

cholera, and Bacillus subtilis (Nanamiya et al., 2008; Das et al., 2009; Gaca et al., 2013). It has also been shown that small amounts of (p)ppGpp produced in a leaky, Tet-controlled hydrolase-dead rel in $M$. tuberculosis was sufficient to restore the survival phenotype of $\Delta$ rel mutants in a mouse model (Weiss and Stallings, 2013). Although the bifunctional Rel is the principal enzyme responsible for the rapid accumulation of (p)ppGpp upon nutrient starvation, the basal level of (p)ppGpp provided by MS_RHII-RSD may be sufficient for survival of $M$. smegmatis $\Delta$ rel mutant under nutrient starvation in the absence of Rel. The intact hydrolase domain left in the rel synthetase-dead mutant constructed by Dahl et al. (2005) might have reduced the basal (p)ppGpp level generated by MS_RHII-RSD, resulting in the reported loss of viability under starvation. In addition, it is interesting to note that deletion of the (p)ppGpp hydrolase domain alone has been shown to be lethal in E. coli, V. cholera, and M. tuberculosis in the presence of a functional (p)ppGpp synthetase (An et al., 1979; Nanamiya et al., 2008; Das et al., 2009; Weiss and Stallings, 2013). The fact that deletion of Rel which contains the only known hydrolase in $M$. smegmatis is not fatal suggests that there might exist an alternative pathway of (p)ppGpp degradation in M. smegmatis.

\section{Stringent Response vs. Morphological Differentiation}

The involvement of the stringent response in morphological differentiation, mediated by a bifunctional Rel, has been reported for various differentiating bacterial species. Loss of rel blocks 
the initiation of the endosporulation process in $B$. subtilis as well as fruiting body development in Myxococcus xanthus (Ochi et al., 1981; Harris et al., 1998). In Streptomyces coelicolor, which belongs to the same Actinobacteria phylum as mycobacteria, deletion of rel did not affect vegetative growth but formation of exospores (Hesketh et al., 2007). The exact mechanism of how Rel controls the early SMRC/LARC developmental process remains to be determined. In Streptomyces, the (p)ppGppmediated effect on differentiation is thought to work via reduction of the cellular GTP concentration. In Streptomyces rel loss-of-function strains, the GTP level is not reduced and differentiation is blocked (Ochi, 1987). To determine whether GTP depletion may play a role for mycobacterial differentiation, we attempted to prevent GTP depletion by adding guanosine or GTP (up to $2 \mathrm{mM}$ ) to gently starved M. smegmatis cultures. However, the bacilli still formed SMRCs. The complementary experiment trying to restore the SMRC formation in starved $M$. smegmatis $\Delta$ rel by lowering the GTP level was also not conclusive: Exposure of starved M. smegmatis $\Delta$ rel cultures to decoyinine, a GMP synthetase inhibitor (Mitani et al., 1977), to block GTP biosynthesis and thus lower GTP levels, did not generate SMRCs (data not shown). However, we cannot rule out the potential role of GTP in the (p)ppGpp-mediated differentiation process as guanosine and GTP might not penetrate the mycobacterial cell envelope and decoyinine might not be able to inhibit mycobacterial GMP synthetase.

Intriguingly, some key genes found to be upregulated during development of SMRCs and LARCs (Figure 5B, left panel; $\mathrm{Wu}$ et al., under review) appear to be under either direct or indirect control of Rel as summarized in Figure 5B (right panel). The transcript level of $f t s Z$, which encodes the septum formation protein, has been shown to be significantly down-regulated in rel-disrupted strains of M. smegmatis (Gupta et al., 2016). Likewise, the expression of two other septum-formation components FtsW and PBPB was four-fivefolds less in a $M$. tuberculosis rel deletion strain compared to the wild-type strain under starvation (Dahl et al., 2003). Moreover, the DNA condensation protein Hlp, showed a decreased transcript level in a $M$. tuberculosis rel deletion strain (Dahl et al., 2003). Reduced expression of these genes in the absence of Rel might explain the failure of SMRC/LARC formation, including the absence of nucleoid compaction observed in our $\Delta$ rel mutant. Another appealing phenotype observed in our $M$. smegmatis $\Delta$ rel mutant is the absence of intracellular lipid bodies (proposed to be composed of triacylglycerol; Garton et al., 2002; Daniel et al., 2004) in gently starved bacilli. Although no key genes involved in triacylglycerol biosynthesis and hydrolysis (e.g., tgs 1 and genes encoding lipases) and downstream fatty acid utilization (e.g., aceA) have been shown to be under direct regulation of Rel in mycobacteria, (p)ppGpp has been suggested to regulate lipid metabolism by inhibiting fatty acid biosynthesis (Heath et al., 1994), but how the stringent response factor mediates the lipid body persistence in SMRCs remains to be determined.

\section{CONCLUSION}

Our rel knockout studies showed that the mycobacterial stringent response factor is required for the formation of both SMRCs and LARCs. Loss of rel function blocks the first cellular differentiation step in the formation of mycobacterial resting cells - the formation of the septated multi-nucleoided cells. Rel is not required for maintaining viability of starved cells. Collectively, this suggests a morphogenetic rather than a survival function for the stringent response factor in M. smegmatis. A low basal level of (p)ppGpp (perhaps produced by MS_RHII-RSD) may be sufficient for survival under starvation, while a high level of (p)ppGpp (requiring functional Rel) may be necessary for morphological differentiation.

\section{AUTHOR CONTRIBUTIONS}

M-LW and TD conceived the project and designed the strategy. CC generated the relA nonsense mutant and carried out the preliminary studies. M-LW carried out all the other experiments. M-LW and TD analyzed the data and wrote the manuscript.

\section{FUNDING}

This work was supported by the Singapore Ministry of Health's National Medical Research Council under its TCR Flagship Grant NMRC/TCR/011-NUHS/2014 to TD and is part of the Singapore Programme of Research Investigating New Approaches to Treatment of Tuberculosis (SPRINT-TB; www.sprinttb.org) led by Nick Paton. M-LW receives a research scholarship from Yong Loo Lin School of Medicine.

\section{ACKNOWLEDGMENTS}

We thank Professor Graham F. Hatfull (University of Pittsburgh) for sharing his recombineering tools (vector pJV62 and pJV53). We acknowledge Professor William R. Jacobs (Albert Einstein College of Medicine) for the gift of the plasmids pYUB854 and pMV306. We would also like to thank Lee Shu Ying and Zhang Weian from Confocal Microscopy Unit (National University of Singapore) for their assistance with confocal microscopy.

\section{SUPPLEMENTARY MATERIAL}

The Supplementary Material for this article can be found online at: http://journal.frontiersin.org/article/10.3389/fmicb. 2016.01390 


\section{REFERENCES}

An, G., Justesen, J., Watson, R. J., and Friesen, J. D. (1979). Cloning the spoT gene of Escherichia coli: identification of the spoT gene product. J. Bacteriol. 137, 1100-1110.

Avarbock, A., Avarbock, D., Teh, J. S., Buckstein, M., Wang, Z. M., and Rubin, H. (2005). Functional regulation of the opposing (p)ppGpp synthetase/hydrolase activities of RelMtb from Mycobacterium tuberculosis. Biochemistry 44, 99139923. doi: 10.1021/bi0505316

Avarbock, D., Salem, J., Li, L. S., Wang, Z. M., and Rubin, H. (1999). Cloning and characterization of a bifunctional RelA/SpoT homologue from Mycobacterium tuberculosis. Gene 233, 261-269. doi: 10.1016/S0378-1119(99)00114-6

Betts, J. C., Lukey, P. T., Robb, L. C., McAdam, R. A., and Duncan, K. (2002). Evaluation of a nutrient starvation model of Mycobacterium tuberculosis persistence by gene and protein expression profiling. Mol. Microbiol. 43, 717731. doi: 10.1046/j.1365-2958.2002.02779.x

Boutte, C. C., and Crosson, S. (2013). Bacterial lifestyle shapes stringent response activation. Trends Microbiol. 21, 174-180. doi: 10.1016/j.tim.2013.01.002

Braeken, K., Moris, M., Daniels, R., Vanderleyden, J., and Michiels, J. (2006). New horizons for (p)ppGpp in bacterial and plant physiology. Trends Microbiol. 14, 45-54. doi: 10.1016/j.tim.2005.11.006

Dahl, J. L., Arora, K., Boshoff, H. I., Whiteford, D. C., Pacheco, S. A., Walsh, O. J., et al. (2005). The relA homolog of Mycobacterium smegmatis affects cell appearance, viability, and gene expression. J. Bacteriol. 187, 2439-2447. doi: 10.1128/JB.187.7.2439-2447.2005

Dahl, J. L., Kraus, C. N., Boshoff, H. I., Doan, B., Foley, K., Avarbock, D., et al. (2003). The role of RelMtb-mediated adaptation to stationary phase in longterm persistence of Mycobacterium tuberculosis in mice. Proc. Natl. Acad. Sci. U.S.A. 100, 10026-10031. doi: 10.1073/pnas.1631248100

Dalebroux, Z. D., and Swanson, M. S. (2012). ppGpp: magic beyond RNA polymerase. Nat. Rev. Microbiol. 10, 203-212. doi: 10.1038/nrmicro2720

Daniel, J., Deb, C., Dubey, V. S., Sirakova, T. D., Abomoelak, B., Morbidoni, H. R., et al. (2004). Induction of a novel class of diacylglycerol acyltransferases and triacylglycerol accumulation in Mycobacterium tuberculosis as it goes into a dormancy-like state in culture. J. Bacteriol. 186, 5017-5030. doi: 10.1128/jb.186.15.5017-5030.2004

Das, B., Pal, R. R., Bag, S., and Bhadra, R. K. (2009). Stringent response in Vibrio cholerae: genetic analysis of spoT gene function and identification of a novel (p)ppGpp synthetase gene. Mol. Microbiol. 72, 380-398. doi: 10.1111/j.13652958.2009.06653.x

Gaca, A. O., Kajfasz, J. K., Miller, J. H., Liu, K., Wang, J. D., Abranches, J., et al. (2013). Basal levels of (p)ppGpp in Enterococcus faecalis: the magic beyond the stringent response. MBio 4:e00646-13. doi: 10.1128/mBio.00646-13

Garton, N. J., Christensen, H., Minnikin, D. E., Adegbola, R. A., and Barer, M. R. (2002). Intracellular lipophilic inclusions of mycobacteria in vitro and in sputum. Microbiology 148, 2951-2958. doi: 10.1099/00221287-148-10-2951

Gengenbacher, M., Rao, S. P., Pethe, K., and Dick, T. (2010). Nutrient-starved, nonreplicating Mycobacterium tuberculosis requires respiration, ATP synthase and isocitrate lyase for maintenance of ATP homeostasis and viability. Microbiology 156, 81-87. doi: 10.1099/mic.0.033084-0

Gupta, K. R., Baloni, P., Indi, S. S., and Chatterji, D. (2016). Regulation of growth, cell shape, cell division, and gene expression by second messengers (p)ppGpp and cyclic Di-GMP in Mycobacterium smegmatis. J. Bacteriol. 198, 1414-1422. doi: 10.1128/JB.00126-16

Harris, B. Z., Kaiser, D., and Singer, M. (1998). The guanosine nucleotide (p)ppGpp initiates development and A-factor production in Myxococcus xanthus. Genes Dev. 12, 1022-1035. doi: 10.1101/gad.12.7.1022

Hauryliuk, V., Atkinson, G. C., Murakami, K. S., Tenson, T., and Gerdes, K. (2015). Recent functional insights into the role of (p)ppGpp in bacterial physiology. Nat. Rev. Microbiol. 13, 298-309. doi: 10.1038/nrmicro3448

Heath, R. J., Jackowski, S., and Rock, C. O. (1994). Guanosine tetraphosphate inhibition of fatty acid and phospholipid synthesis in Escherichia coli is relieved by overexpression of glycerol-3-phosphate acyltransferase (plsB). J. Biol. Chem. 269, 26584-26590.

Hesketh, A., Chen, W. J., Ryding, J., Chang, S., and Bibb, M. (2007). The global role of ppGpp synthesis in morphological differentiation and antibiotic production in Streptomyces coelicolor A3(2). Genome Biol. 8:R161. doi: 10.1186/gb-2007-88-r161
Jain, V., Saleem-Batcha, R., China, A., and Chatterji, D. (2006). Molecular dissection of the mycobacterial stringent response protein Rel. Protein Sci. 15, 1449-1464. doi: 10.1110/ps.062117006

Kaser, M., Ruf, M. T., Hauser, J., Marsollier, L., and Pluschke, G. (2009). Optimized method for preparation of DNA from pathogenic and environmental mycobacteria. Appl. Environ. Microbiol. 75, 414-418. doi: 10.1128/AEM.01358-08

Klinkenberg, L. G., Lee, J. H., Bishai, W. R., and Karakousis, P. C. (2010). The stringent response is required for full virulence of Mycobacterium tuberculosis in guinea pigs. J. Infect. Dis. 202, 1397-1404. doi: 10.1086/656524

Loebel, R. O., Shorr, E., and Richardson, H. B. (1933a). The influence of adverse conditions upon the respiratory metabolism and growth of human tubercle bacilli. J. Bacteriol. 26, 167-200.

Loebel, R. O., Shorr, E., and Richardson, H. B. (1933b). The influence of foodstuffs upon the respiratory metabolism and growth of human tubercle bacilli. J. Bacteriol. 26, 139-166.

Mathew, R., Ojha, A. K., Karande, A. A., and Chatterji, D. (2004). Deletion of the rel gene in Mycobacterium smegmatis reduces its stationary phase survival without altering the cell-surface associated properties. Curr. Sci. 86, 149-153.

Mitani, T., Heinze, J. E., and Freese, E. (1977). Induction of sporulation in Bacillus subtilis by decoyinine or hadacidin. Biochem. Biophys. Res. Commun. 77, 1118-1125. doi: 10.1016/S0006-291X(77)80094-6

Morita, R. Y. (1993). "Bioavailability of energy and the starvation state," in Starvation in Bacteria, ed. S. Kjelleberg (Boston, MA: Springer), 1-23.

Murdeshwar, M. S., and Chatterji, D. (2012). MS_RHII-RSD, a dual-function RNase HII-(p)ppGpp synthetase from Mycobacterium smegmatis. J. Bacteriol. 194, 4003-4014. doi: 10.1128/JB.00258-12

Nanamiya, H., Kasai, K., Nozawa, A., Yun, C. S., Narisawa, T., Murakami, K., et al. (2008). Identification and functional analysis of novel (p)ppGpp synthetase genes in Bacillus subtilis. Mol. Microbiol. 67, 291-304. doi: 10.1111/j.13652958.2007.06018.x

Nyka, W. (1974). Studies on the effect of starvation on mycobacteria. Infect. Immun. 9, 843-850.

Ochi, K. (1987). Changes in nucleotide pools during sporulation of Streptomyces griseus in submerged culture. Microbiology 133, 2787-2795. doi: 10.1099/00221287-133-10-2787

Ochi, K., Kandala, J. C., and Freese, E. (1981). Initiation of Bacillus subtilis sporulation by the stringent response to partial amino acid deprivation. J. Biol. Chem. 256, 6866-6875.

Ojha, A. K., Mukherjee, T. K., and Chatterji, D. (2000). High intracellular level of guanosine tetraphosphate in Mycobacterium smegmatis changes the morphology of the bacterium. Infect. Immun. 68, 4084-4091. doi: 10.1128/IAI.68.7.4084-4091.2000

Potrykus, K., and Cashel, M. (2008). (p)ppGpp: still magical? Annu. Rev. Microbiol. 62, 35-51. doi: 10.1146/annurev.micro.62.081307.162903

Primm, T. P., Andersen, S. J., Mizrahi, V., Avarbock, D., Rubin, H., and Barry, C. E. III. (2000). The stringent response of Mycobacterium tuberculosis is required for long-term survival. J. Bacteriol. 182, 4889-4898. doi: 10.1128/JB.182.17.48894898.2000

Rittershaus, E. S., Baek, S. H., and Sassetti, C. M. (2013). The normalcy of dormancy: common themes in microbial quiescence. Cell Host Microbe 13, 643-651. doi: 10.1016/j.chom.2013.05.012

Shi, L., Gunther, S., Hubschmann, T., Wick, L. Y., Harms, H., and Muller, S. (2007). Limits of propidium iodide as a cell viability indicator for environmental bacteria. Cytometry A 71, 592-598. doi: 10.1002/cyto.a.20402

Stent, G. S., and Brenner, S. (1961). A genetic locus for the regulation of ribonucleic acid synthesis. Proc. Natl. Acad. Sci. U.S.A. 47, 2005-2014. doi: 10.1073/pnas.47.12.2005

Tomioka, H. (1983). Purification and characterization of the Tweenhydrolyzing esterase of Mycobacterium smegmatis. J. Bacteriol. 155, 1249-1259.

van Kessel, J. C., and Hatfull, G. F. (2008). Efficient point mutagenesis in mycobacteria using single-stranded DNA recombineering: characterization of antimycobacterial drug targets. Mol. Microbiol. 67, 1094-1107. doi: 10.1111/j.1365-2958.2008.06109.x

van Kessel, J. C., Marinelli, L. J., and Hatfull, G. F. (2008). Recombineering mycobacteria and their phages. Nat. Rev. Microbiol. 6, 851-857. doi: $10.1038 /$ nrmicro2014 
Weiss, L. A., and Stallings, C. L. (2013). Essential roles for Mycobacterium tuberculosis Rel beyond the production of (p)ppGpp. J. Bacteriol. 195, 56295638. doi: 10.1128/JB.00759-13

Wu, M.-L., Gengenbacher, M., and Dick, T. (2016). Mild nutrient starvation triggers the development of a small-cell survival morphotype in mycobacteria. Front. Microbiol. 7:947. doi: 10.3389/fmicb.2016. 00947

Xie, Z., Siddiqi, N., and Rubin, E. J. (2005). Differential antibiotic susceptibilities of starved Mycobacterium tuberculosis isolates. Antimicrob. Agents Chemother. 49, 4778-4780. doi: 10.1128/AAC.49.11.4778-4780.2005
Conflict of Interest Statement: The authors declare that the research was conducted in the absence of any commercial or financial relationships that could be construed as a potential conflict of interest.

Copyright $(02016 \mathrm{Wu}$, Chan and Dick. This is an open-access article distributed under the terms of the Creative Commons Attribution License (CC BY). The use, distribution or reproduction in other forums is permitted, provided the original author(s) or licensor are credited and that the original publication in this journal is cited, in accordance with accepted academic practice. No use, distribution or reproduction is permitted which does not comply with these terms. 